\title{
XÂY DỰNG CƠ SỞ DŨ LIỆ ĐỊA LÝ TỬ DŨ̉ LIỆ ẢNH THU NHậ̣N CỦA THIẾT BỊ BAY KHÔNG NGƯỜI LÁI (UAV)
}

\author{
ThS. ĐÕ VĂN DƯO'NG \\ Trường Đại học Tài nguyên và Môi trường Hà Nội
}

\section{Tóm tắt:}

Trên thế giới thiết bị bay không người lái (UAV) đã được sử dụng rất sớm vào công tác đo ảnh, dữ liệu ảnh của UAV được ứng dụng trong cả lĩnh vực quân sự và dân sự nhu: giám sát biên giới, giám sát thiên tai, theo dõi sản xuất nông nghiệp, giám sát giao

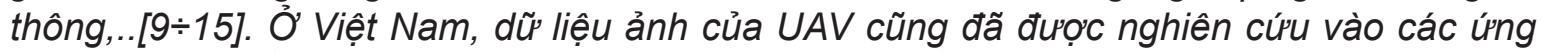

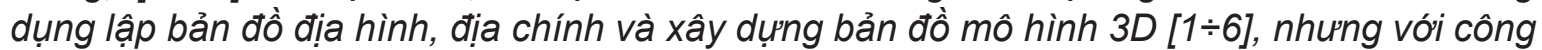
tác xây dựng cơ sở dũ liệu (CSDL) địa lý từ dữ liệu ảnh của thiết bị này thì mới đang được thử nghiệm. Bài viết này nhằm mô tả quá trình xây dựng một cơ sở dữ liệu địa lý từ dữ liệu hình ảnh của UAV và phân tích đánh giá kết quả thực nghiệm.

\section{Giới thiệu chung về UAV và dữ liệu ảnh thu nhận}

Thiết bị bay không người lái (UAV) là thiết bị bay được điều khiển từ xa bán tự động hoặc tự động bằng thiết bị dẫn đường GPS và trên đó gắn thiết bị máy ảnh được các hãng UAV đặt hàng thiết kế riêng cho từng dạng UAV như máy ảnh Olympus EP gắn trên UAV MD-1000 hay máy ảnh Sony NEX5R gắn trên UAV Trimble UX5;... với chế độ chụp ảnh tự động và những thông số kỹ thuật riêng biệt [1]. Các báo cáo kết quả bay thử nghiệm của Cục Bản đồ - Bộ Tổng tham mưu, của Đại học Quốc gia TP. Hồ Chí Minh, ...trên các mẫu UAV khác nhau cho thấy công tác thu nhận và xử lý dữ liệu ảnh của các hệ thống UAV cơ bản theo một quy trình như sau $[1 \div 6]$ :

- Xác định phạm vi bay

- Lập mốc và đo khống chế ảnh ngoại nghiệp

- Thiết kế bay và bay chụp ảnh

- Xử lý ảnh bằng phần mềm chuyên dụng

- Kiểm tra kết quả xử lý ảnh

- Kết quả các sản phẩm tạo ra. (hình 1)

Ngày nhận bài: 25/8/2015
2. Quy trình xử lý và xây dựng CSDL địa lý từ dữ liệu ảnh của UAV

Ảnh thu nhận từ thiết bị UAV có độ phân giải rất cao lên đến $\mathrm{cm}$, do vậy nó có thể xử lý và xây dựng CSDL địa lý ở các tỷ lệ lớn như 1/1000,1/2000, 1/5000. Việc xử lý ảnh được tiến hành trên các phần chuyên dụng xử lý ảnh đi kèm với các thiết bị như phần mềm ObitGIS của thiết bị bay MicroDrone, phần mềm Postflight Terra của thiết bị bay Sensefly,... hoặc trạm xử lý ảnh số Image Station (đã được sử dụng phổ biến ở Việt Nam). Kết quả xử lý ảnh sẽ được chuẩn hóa khuôn dạng dữ liệu địa hình và nhập vào phần mềm GIS (GeoMedia, MapInfo, ArcGIS). Quy trình xử lý và xây dựng CSDL địa lý có thể tổng quát như hình 2.

\section{Thực nghiệm thiết bị bay MD-1000 khu vực xã Vật Lại, Ba Vi, Hà Nội}

\section{1. Đặc điểm khu vực nghiên cứu}

Xã Vật Lại nằm ở phía Tây Thành phố Hà Nội, cách trung tâm huyện Ba Vì khoảng $1,5 \mathrm{~km}$, cách trung tâm Hà Nội khoảng $50 \mathrm{~km}$. Xã nằm ở phía Tây huyện $\mathrm{Ba} V \mathrm{Vì} \mathrm{nơi}$ chuyển tiếp giữa vùng đồi gò bán sơn địa và vùng đồng bằng. Khu vực thực nghiệm bay chụp nằm trong vùng giới hạn bởi các điểm

Ngày chấp nhận đăng: 16/9/2015 

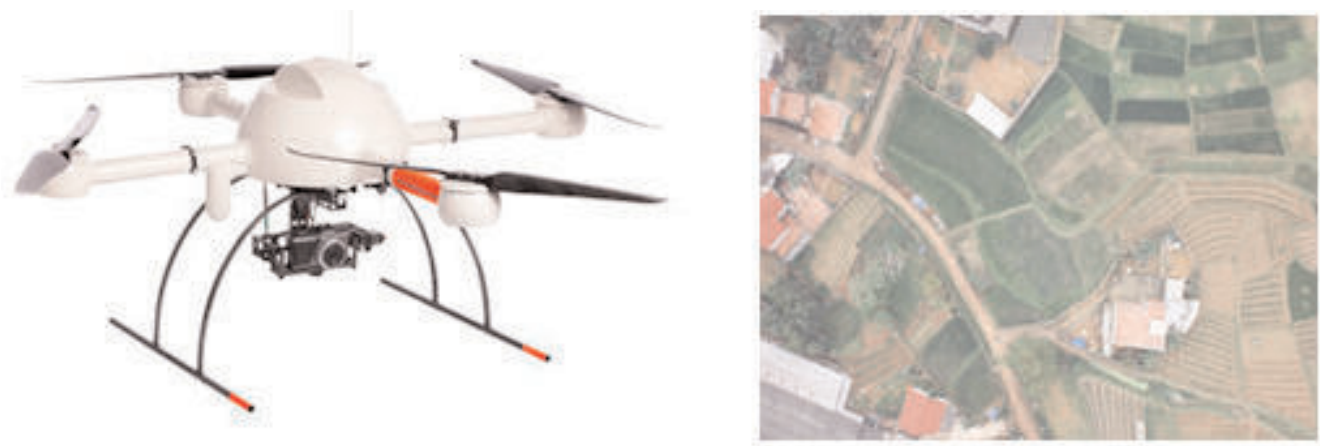

Hình 1: UAV MD4-1000 và dữ liệu hình ảnh thu nhận từ hệ thống

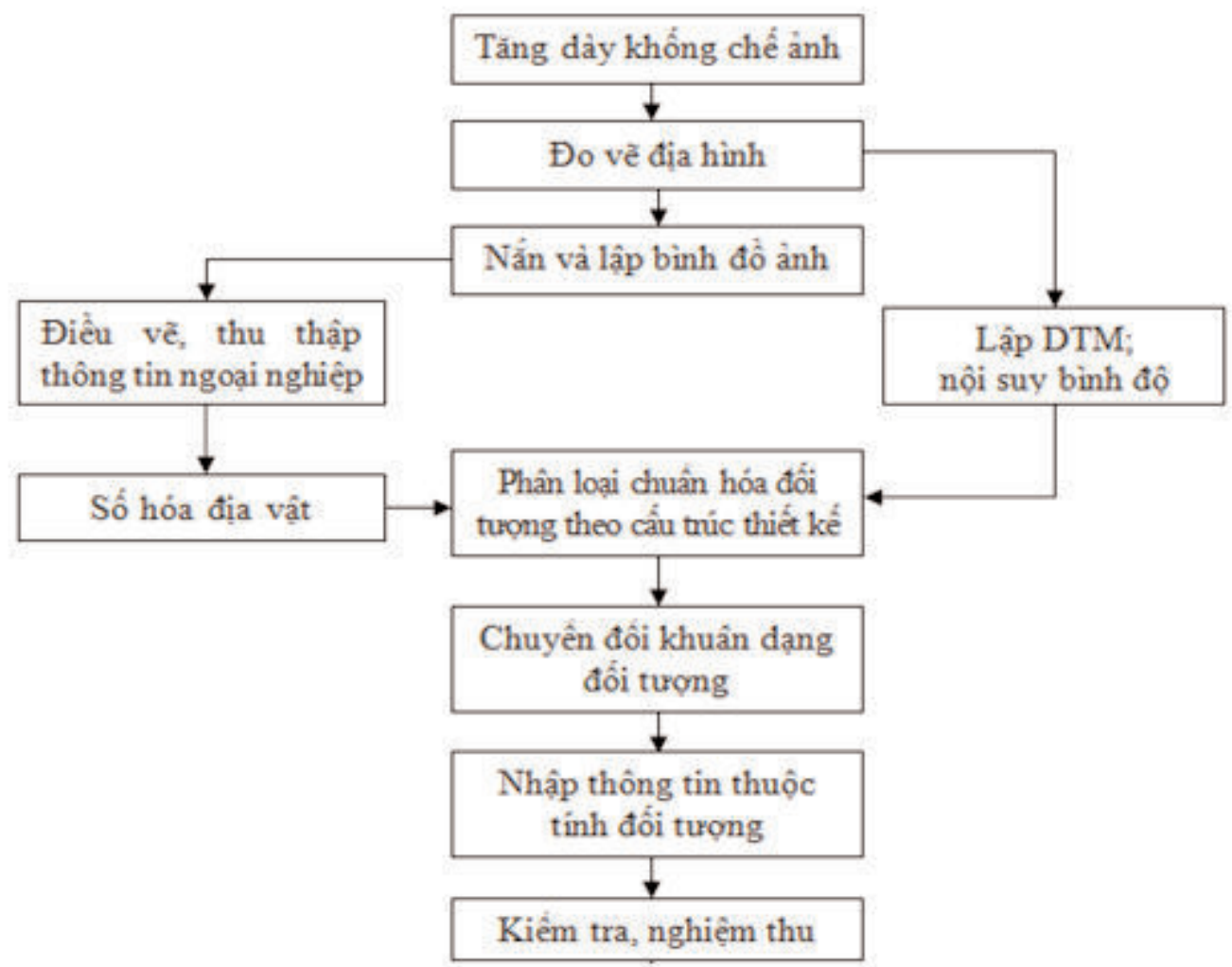

Hình 2: Quy trình xử lý và xây dựng CSDL địa lý từ dữ liệu ảnh của UAV

$1,2,3,4$ có tọa độ địa lý $1\left(21^{0} 12^{\prime} 41^{\prime \prime}, \quad\right.$ Tên máy chụp: Olympus EP $\left.105^{\circ} 23^{\prime} 21^{\prime \prime}\right)$; 2(21012'35", $\left.105^{\circ} 23^{\prime 2} 22^{\prime \prime}\right)$; Tiêu cự: $17.090476 \mathrm{~mm}$ $3\left(21^{0} 12^{\prime} 32^{\prime \prime}, \quad 105^{\circ} 23^{\prime} 11^{\prime \prime}\right)$ và $4\left(21^{0} 12^{\prime} 37^{\prime \prime}, \quad\right.$ Cỡ ảnh $(C C D)$ : $17.7408 \times 13.3056 m m$ $\left.105^{\circ} 23^{\prime} 09^{\prime \prime}\right)$ thuộc thôn Yên Bồ - xã Vật Lại [7]. (hình 3)

3.2. Các thông số thiết đặt máy chụp ảnh, ảnh chụp và máy bay

Kích thước pixel: $4.4 \mu \mathrm{m}$

Tọa độ điểm chính ảnh (PBS): $X=0.032982 \mathrm{~mm}, Y=-0.074 \mathrm{~mm}$ 
Tham số méo hình hướng tâm: $\mathrm{K} 1=1.679973 \mathrm{E}-4 ; \quad \mathrm{K} 2=-3.286605 \mathrm{E}-7$

Tham số méo hình tiếp tuyến: $\mathrm{P} 1=-3.124259 \mathrm{E}-5 \quad \mathrm{P} 2=5.29212 \mathrm{E}-5$

Tên thiết bị bay: Microdrones MD4-1000, có hỗ trợ hệ thống GPS/IMU trên thiết bị bay.

Độ cao chụp ảnh so với mặt đất: $100 \mathrm{~m}$

Độ phủ dọc: $80 \%$

Độ phủ ngang: 60\%

Tỷ lệ chụp ảnh: 1/5800.

3.3. Kết quả bay chụp và đo khống chế

- Số lượng ảnh bay chụp: 75 ảnh.

- Diện tích bay chụp: 8.7 ha.
- Đo khống chế ảnh ngoại nghiệp: 08 điểm. (hình 4)

Chất lượng ảnh chụp có độ nét cao $0.02 \mathrm{~m}$, màu sắc trung thực, khả năng nhận dạng rất tốt.

Các điểm khống chế ảnh được chọn ở những vị trí rõ nét, thông thoáng dễ dàng cho việc đo đạc tọa độ độ cao.

3.4. Các bước thực nghiệm xử lý ảnh và xây dựng CSDL địa lý

\subsubsection{Tăng dày khống chế ảnh}

Sử dụng phần mềm xử lý Image Station Automatic Triangulation (ISAT). công.

Công tác đo ảnh kết hợp tự động và thủ

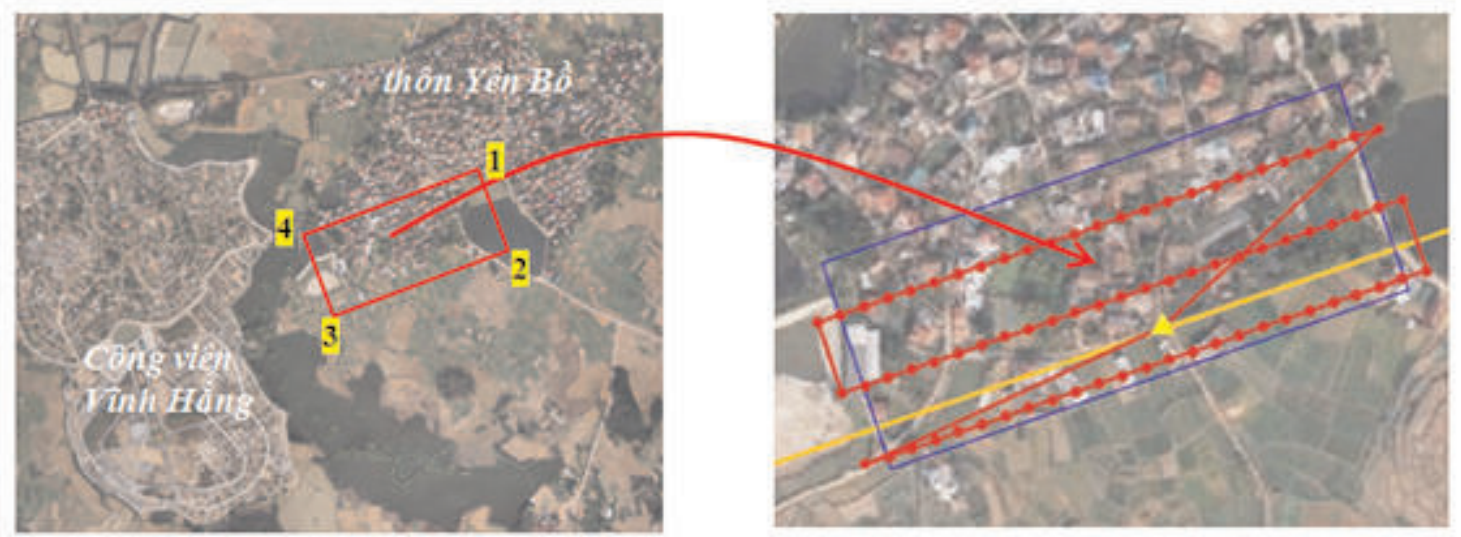

Hình 3: Vị trí địa lý khu vực bay thử nghiệm thu nhận ảnh của UAV
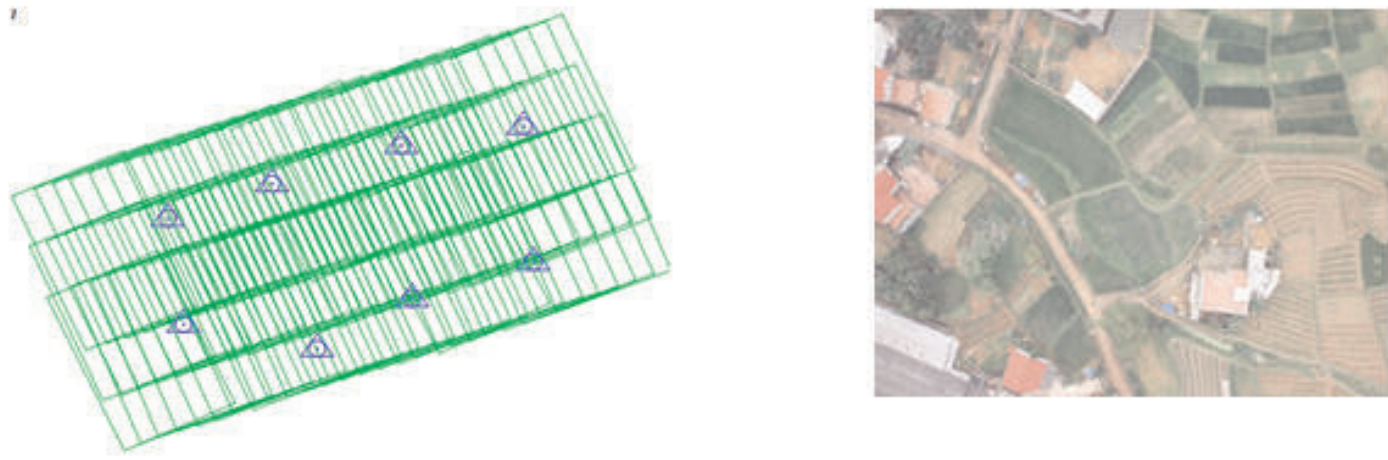

Hình 4: Mô tả dải bay, vị trí điểm KCANN và hình ảnh thu nhận từ UAV 
Kết quả tăng dày:

- Số tuyến bay: 3

- Số lượng ảnh: 75

- Số điểm khống chế: 06

- Số điểm kiểm tra: 02

- Sai số trung phương đo ảnh: 9.4 m

- Sai số tồn tại tại điểm khống chế ảnh: Mặt phẳng: $0.050 \mathrm{~m}$; Độ cao: $0.038 \mathrm{~m}$

- Sai số tồn tại tại điểm kiểm tra: Mặt phẳng: $0,083 \mathrm{~m}$; Độ cao: $0,077 \mathrm{~m}$.

Đối chiếu các tiêu chuẩn kỹ thuật trong Tiêu chuẩn ngành [8] và độ phân giải hình ảnh thu nhận đạt tới $0.02 \mathrm{~m}$, ta khẳng định kết quả tăng dày khống chế ảnh đủ độ chính xác để tiến hành xây dựng dữ liệu địa lý với mức độ chi tiết tương đương bản đồ địa hình tỷ lệ 1/1000.

\subsection{2. Đo vẽ địa hình}

Sử dụng modul ISSD, ISDC, ISFC của trạm đo vẽ ảnh số ImageStation để đo vẽ địa hình và các đối tượng địa vật.

\section{a. Đo vẽ mô tả địa hình}

Đặc điểm địa hình của khu vực Vật Lại, Ba Vì là địa hình chuyển đổi giữa vùng đồi gò bán sơn địa và vùng đồng bằng, là vùng đồi thấp xen kẽ ruộng bậc thang có chênh cao nhỏ, có vài vùng thực phủ xen kẽ dạng cây trồng lâu năm. Do địa hình hầu như

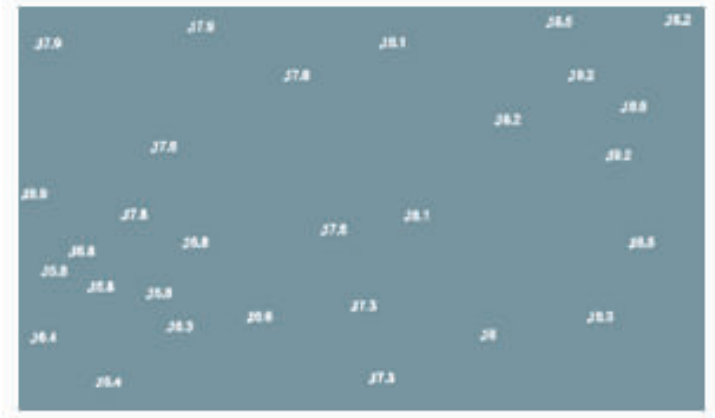

Hình 5: Kết quả đo điểm độ cao mô tả địa hình không có nếp đứt gãy nên chủ yếu sử dụng dạng điểm độ cao để mô tả mặt đất. Các vùng thực phủ có kích thước khá nhỏ và do ảnh chụp ở độ cao thấp, có thể nhìn thấy nhiều khe hở nên có thể cắt tiêu đo $100 \%$ trên mặt đất mà không phải áp dụng biện pháp trừ chiều cao cây. Dữ liệu điểm độ cao sẽ kết hợp với dữ liệu đo vẽ giao thông, thủy hệ đủ để tạo mô hình số độ cao với độ chi tiết cao nhất (Hình 5).

\section{b. Đo vẽ giao thông, thủy hệ}

Đường giao thông trong khu vực bay chụp chủ yếu là đường trong làng nên chỉ cần thể hiện lề đường và lòng đường nếu có, khu vực này không có sông suối mà chỉ có rãnh thoát nước và một số ao hồ. Thể hiện các ao hồ bằng cách vẽ đối tượng dạng vùng dọc theo đường mép nước cũng chính là đường bờ nước (Hình 6, Hình 7).

(Xem hinh 5, 6, 7, 8)

\subsubsection{Lập mô hình số địa hình (DTM), nội suy binh độ}

Lập mô hình DTM được tiến hành trên modul ISAE của hệ thống phần mềm ImageStation. Các điểm độ cao và các đối tượng giao thông, thủy hệ được đưa vào công tác lập DTM, độ chính xác mô hình số địa hình DTM đạt được nhỏ hơn $0.3 \mathrm{~m}$.

Sau khi có mô hình bề mặt, tiến hành công tác nội suy đường bình độ với khoảng

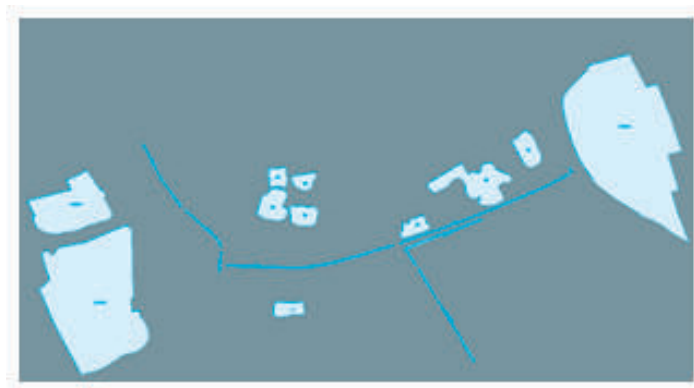

Hình 7: Kết quả đo vẽ hệ thống thủy hệ 
cao đều $0.5 \mathrm{~m}$ (Hình 8). Việc nội suy bình độ có thể thực hiện trên modul ISEE của trạm ảnh số ImageStation.

\subsubsection{Nắn và lập bình đồ ảnh}

Nắn ảnh được thực hiện trên modul ISOP của phần mềm ImageStation. Sau khi nắn các tấm ảnh sẽ được cắt và ghép trên modul OrthoPro với sai số ghép ảnh nhỏ hơn 0.6m. (hình 9)

\subsection{5. Điều vẽ, thu thập thông tin ngoại nghiệp}

Quá trình này được tiến hành với mục đích điều tra, xác định các yếu tố định tính, định lượng của các đối tượng, các nội dung

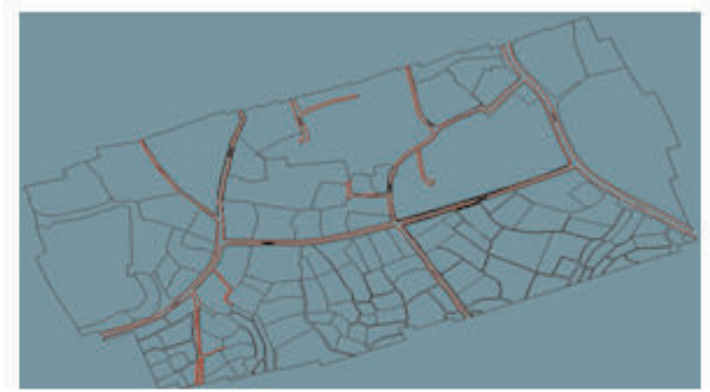

Hình 6: Kết quả đo vẽ hệ thống giao thông ghi chú trên bản đồ như: tính chất của hệ thống thuỷ hệ, giao thông, dân cư, địa lý, hành chính...

Kết quả điều vẽ phải được vẽ lên bình đồ ảnh theo quy định của Qui phạm và phục vụ việc số hóa bổ sung địa vật và nhập thông tin CSDL địa lý cho đối tượng. Những đối tượng cần thiết nhưng chưa có ký hiệu quy định thì phải thể hiện theo đúng hình dáng, tại đúng vị trí kèm theo ghi chú giải thích. Việc lấy, bỏ, tổng hợp và xê dịch phải biểu thị tuân theo nguyên tắc: đối tượng thứ yếu nhường cho đối tượng chủ yếu, đối tượng có yêu cầu độ chính xác thấp nhường cho đối tượng có yêu cầu độ chính xác cao.

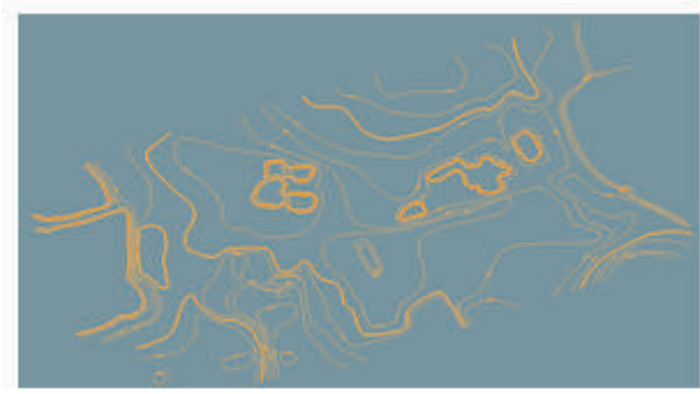

Hình 8: Kết quả nội suy đường bình độ

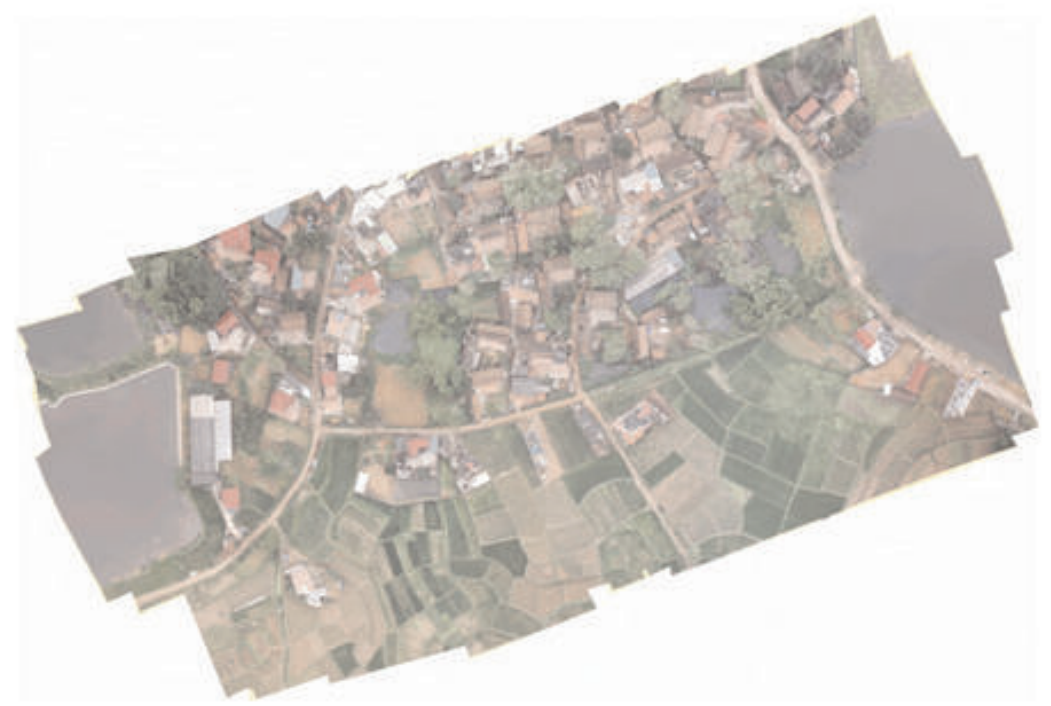

Hình 9: Kết quả nắn và ghép ảnh trực giao khu đo 


\subsubsection{Số hóa địa vật}

Sau khi đo vẽ các yếu tố địa hình, giao thông và thủy hệ trên trạm ảnh số, các yếu tố nội dung bản đồ còn lại được số hóa trên nền trực ảnh theo trình tự:

- Các đối tượng liên quan giao thông;

- Các đối tượng liên quan thủy hệ;

- Dân cư và các đối tượng văn hóa, kinh tế, xã hội;

- Ranh giới hành chính;

- Thực vật.

Do ảnh có kích thước quá nhỏ, rất nhiều địa vật bị phân chia nằm trên nhiều mô hình khác nhau, vì thế cùng một địa vật phải vẽ trên nhiều mô hình và xảy ra vấn đề tiếp khớp về mặt bằng, độ cao và phát sinh công việc tạo vùng.

3.4.7. Phân loại và chuẩn hoá đối tượng địa lý theo cấu trúc thiết kế

*Phân loại các đối tượng địa lý theo cấu trúc đã được thiết kế

Phân loại các đối tượng là công việc tách các đối tượng theo các nhóm trong quy định xây dựng CSDL địa lý, bao gồm 10 nhóm: điểm khống chế, địa giới hành chính, địa danh, địa hình, thủy văn, giao thông, nhóm bề mặt sử dụng, nhóm ranh giới sử dụng, nhóm hạ tầng kỹ thuật và nhóm hạ tầng dân cư.

\section{* Chuẩn hóa các đối tượng địa lý}

Sau khi phân loại xong tiến hành chuẩn hóa dữ liệu, gồm:

+ Chuẩn hóa về mặt phân loại đối tượng địa lý nhằm đảm bảo độ chính xác chất lượng dữ liệu;

+ Chuẩn hóa loại đối tượng: chuẩn hóa đối tượng dạng điểm, dạng đường, dạng vùng. Trong lớp đối tượng dạng điểm phải không được lẫn các đối tượng là đường và vùng, tương tự như vậy với lớp đường và vùng;

+ Chuẩn hóa về quan hệ hình học như: các đường bình độ không được cắt nhau, các vùng trong một lớp không được chồng đè hoặc có khoảng hở, ...

\subsubsection{Chuyển đổi khuôn dạng của dữ liệu}

Sau khi phân loại và chuẩn hóa xong, tiến hành chuyển đổi khuôn dạng dữ liệu sang khuôn dạng CSDL địa lý (Geodatabase) của ArcGIS. Các bước tiến hành như sau:

- Khởi động phần mềm Visio và mở file cấu trúc CSDL địa lý GIS đã được tạo rồi xuất (export) từ khuôn dạng của phần mềm Visio sang XML.

- Khởi động phần mềm ArcCatalog rồi nhập (import) file XML vừa được tạo ở bước trên vào khuôn dạng Geodatabase của phần mềm ArcGIS (sử dụng công cụ Case Schema Creation trên phần mềm ArcCatalog). Ta sẽ được một Geodatabase với 10 lớp dữ liệu (FeatureDataset) theo đúng thiết kế. (hình 10)

- Load dữ liệu của 9 nhóm lớp đã được phân loại và chuẩn hóa trên khuôn dạng *.dgn của MicroStation vào cấu trúc CSDL nền đã được tạo sã̃n trên ArcGIS. Mở từng FeatureDataset và từng lớp cần Load, kích chuột phải vào lớp cần Load rồi vào Loadl Load Data, chọn đường dẫn tới file dữ liệu đầu vào rồi thực hiện các lệnh tiếp theo xuất hiện trên giao diện. (hình 11)

- Riêng với nhóm Địa danh tiến hành chuyển đổi từ khuôn dạng của MicroStatio sang ArcGIS bằng công cụ trên ArcToolbox. Vào ArcToolbox $\rightarrow$ To Geodatabase $\rightarrow$ Import CAD Annotation rồi chọn đường dẫn tới file dũ liệu địa danh đã được phân loại và chuẩn hóa trên MicroStation.

\subsubsection{Nhập thông tin thuộc tính}

Sau khi load dữ liệu từ MicroStation sang 
ArcGIS, tất cả các đối tượng có thuộc tính tính cho các trường còn lại mà các đối trong trường phân nhóm và phân loại đối tượng không thể Domain hóa giá trị tự tượng đã được Domain hóa sẽ nhận giá trị. động. Ví dụ trường "Tên sông suối" phải

Như vậy ta chỉ cần nhập thông tin thuộc nhập thủ công cho từng đối tượng. (hình 12)

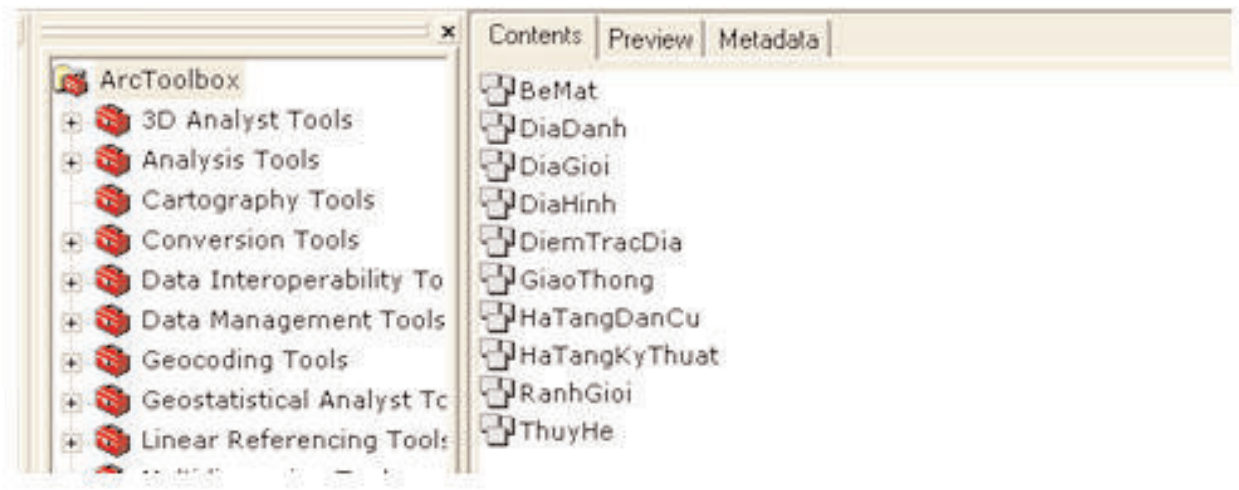

Hình 10: Thiết kế cấu trúc của CSDL địa lý với 10 lớp dữ liệu.

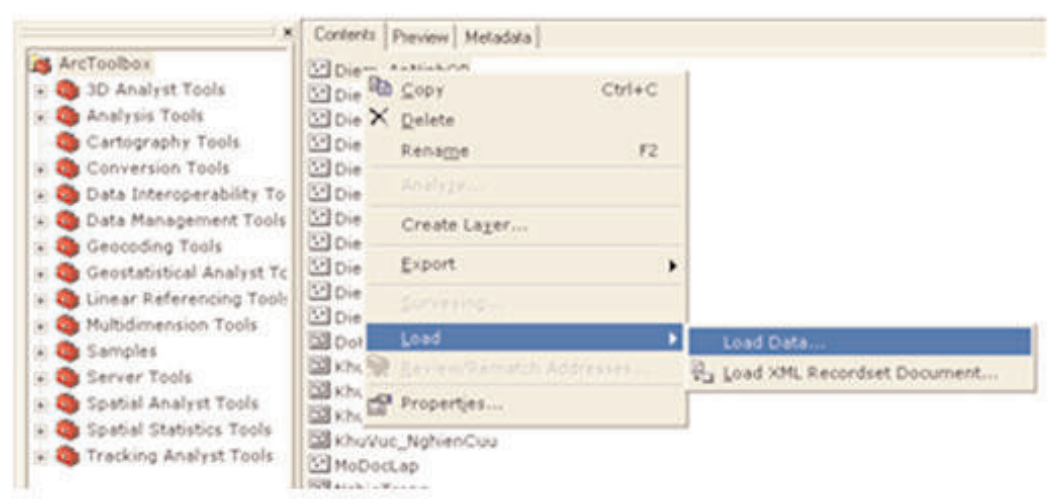

Hình 11: Load dũ liệu từ MicroStation vào cấu trúc đã thiết kế trên ArcGIS

\begin{tabular}{|c|c|c|c|}
\hline Table & & & $x$ \\
\hline 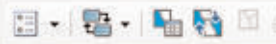 & $5 x$ & & \\
\hline Dữung nởi bọ dang đường & & & $x$ \\
\hline Loại đường nội bộ & Shape_Length & Loại hiện trạng sừ dung & - \\
\hline DuongBoRuong & 18.748493 & DangSuDung & \\
\hline DuongBoRuong & 45.393355 & DangSuDung & \\
\hline DuongBoRuong & 13.997921 & DangSuDung & \\
\hline DuongBoRuong & 75.615677 & DangSuDung & \\
\hline DuongBoRuong & 15.500476 & DangSuDung & \\
\hline DuongBoRuong & 25.431484 & DangSuDung & \\
\hline DuongBoRuong & 5.496754 & DangSuDung & \\
\hline DuongBoRuong & 12.610947 & DangSuDung & \\
\hline Runmenendingn & 71. annกRA & nannciunume & \\
\hline (1) & $\pi$ & , & \\
\hline $14.23,11$ & 圆四 $(0$ out & 261 Selected) & \\
\hline Đường nội bộ dạng đường & & & \\
\hline
\end{tabular}

Hình 12: Nhập dữ liệu thuộc tính trên ArcGIS 


\subsubsection{Kiểm tra nghiệm thu}

- Kiểm tra mức độ đầy đủ của sản phẩm so với dữ liệu gốc và các bảng thuộc tính đối tượng.

- Kiểm tra độ chính xác CSDL không gian và CSDL thuộc tính của sản phẩm so với dữ liệu gốc và các phiếu thuộc tính đối tượng địa lý.

- Kiểm tra quan hệ hình học của các đối tượng trong một lớp và với lớp khác.

Khi kiểm tra, chúng ta sử dụng cả kiểm tra thủ công và kiểm tra tự động sử dụng các công cụ của phần mềm ArcGIS.

\subsection{Kết quả}

Kết hợp với công tác điều tra, thu thập thông tin ngoại nghiệp cùng với việc xử lý ảnh đạt độ chính xác cao (đã trình bày trong mục 3.4.1) và độ phân giải ảnh của thiết bị UAV đạt tới centimet. Do vậy, kết quả xử lý dữ liệu ảnh của UAV đủ độ tin cậy để xây dựng CSDL địa lý với khoảng cao đều địa hình $0.5 \mathrm{~m}$. (hình 13)

\section{Kết luận}

Sử dụng dữ liệu ảnh thu nhận từ thiết bị UAV để xây dựng cơ sở dữ liệu địa hình sẽ là xu hướng mới của ngày nay do chúng có những ưu điểm như:

Chất lượng ảnh tốt và độ phân giải cao (cỡ vài $\mathrm{cm}$ ) đủ độ chính xác theo yêu cầu thông tin để xây dựng CSDL địa lý .

Do thiết bị UAV bay chụp ở độ cao thấp nên hình ảnh các đối tượng rõ nét phục vụ tốt cho việc đo vẽ trên máy tính, giảm bớt rất nhiệu công điều vẽ ngoại nghiệp.

Tuy nhiên việc ứng dụng ảnh thu nhận từ thiết bị UAV để phục vụ CSDL địa lý cũng có một số hạn chế nhất định: Kích thước ảnh bé nên xử lý ảnh với số lượng mô hình rất lớn; vẫn còn có một số ảnh thu nhận được bị nhòe gây khó khăn cho việc số hóa; còn biến dạng hình ảnh do chiều cao của các địa vật gây ra vẫn còn tồn tại.

Như vậy, dữ liệu ảnh thu nhận từ thiết bị UAV hoàn toàn có thể sử dụng cho công tác xây dựng CSDL địa lý với mức độ chi tiết tương đương với bản đồ địa hình tỷ lệ 1/1000. Công nghệ bay chụp UAV sẽ rất

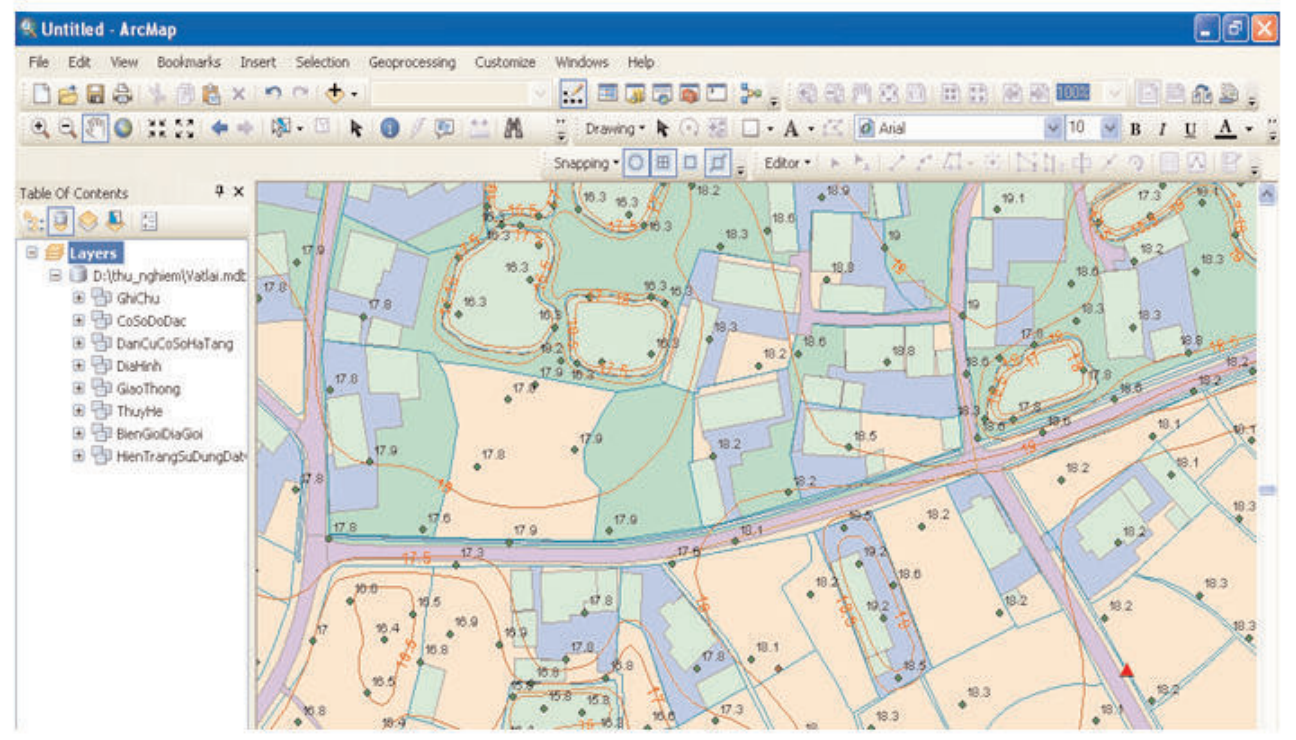

Hình 13: Kết quả xây dựng CSDL địa lý với mức độ chi tiết địa hình tỷ lệ 1:1000 khu vực Vật Lại, Ba Vì từ ảnh của UAV MD-1000 trên phần mềm ArcGIS 
hiệu quả khi sử dụng tại những vùng xa xôi hẻo lánh và khó khăn phức tạp do việc cất và hạ cánh của thiết bị bay đơn giản không cần không gian quá rộng. $\bigcirc$

\section{Tài liệu tham khảo}

[1]. Đào Ngọc Long, Nghiên cứu ứng dụng công nghệ thành lập bản đồ (địa hình và địa chính) tỷ lệ 1/2000 từ ảnh chụp bằng máy chụp ảnh số phổ thông lắp trên máy bay không người lái M100-CT điều khiển bằng sóng Radio. Đề tài cấp Bộ, năm 2014.

[2]. Phan Thị Anh Thư, Lê Văn Trung. Thu nhận ảnh bằng máy bay không người lái phục vụ công tác thành lập bản đồ. Hội thảo ứng dụng GIS toàn quốc năm 2011.

[3]. Xí nghiệp bay chụp ảnh hàng không. Báo cáo kết quả thử nghiệm xử lý ảnh chụp từ thiết bị bay không người lái MD4-1000. Công ty TNHH MTV Trắc địa-Bản đồ, Cục Bản đồ, Bộ Tổng Tham mưu. Tháng 9-2011

[4]. Lê Đại Ngọc, Hoàng Văn Anh. Úng dụng thiết bị bay không người lái Microdrone MD4-1000 trong thành lập bản đồ 3D-độ chính xác cao. Tuyển tập báo cáo Hội nghị khoa học ngành Địa hình quân sự. Tháng $9-2014$.

[5]. Lều Huy Nam. Nghiên cứu thiết bi bay không người lái Trimble UX5 và khả năng ứng dụng trong công tác thành lập bản đồ. Tuyển tập báo cáo Hội nghị khoa học ngành Địa hình quân sự. Tháng 9 - 2014.

[6]. Vũ Phan Long, Lê Thắng. Thử nghiệm thiết bị bay không người lái thành lập mô hình 3D hành lang tuyến điện. Tuyển tập báo cáo Hội nghị khoa học ngành Địa hình quân sự. Tháng 9 - 2014.

\section{[7]. http://www.googlemap.com}

[8]. Tiêu chuẩn ngành "Quy phạm thành lập bản đồ địa hình tỷ lệ 1/500, 1/1000, 1/2000, 1/5000, 1/10000 và 1/25000" (Phần trong nhà). Cục Đo đạc và Bản đồ nhà nước, 96TCN-90.
[9]. Henri Eisenbeiss, A mini unmanned aerial vehicle (UAV): System overview and image acquisition, International Workshop on "PROCESSING AND VISUALIZATION USING HIGH-RESOLUTION IMAGERY" 18-20 November 2004, Pitsanulok, Thailand.

[10]. LIN Zongjian, UAV for mapping-low altitude photogrammetric survey, The International Archives of the Photogrammetry, Remote Sensing and Spatial Information Sciences. Vol. XXXVII. Part B1. Beijing 2008.

[11]. G. J. Grenzdörffer, A. Engel, B. Teichert, The photogrammetric potential of low-cost UAVs in forestry and agriculture, The International Archives of the Photogrammetry, Remote Sensing and Spatial Information Sciences. Vol. XXXVII. Part B1. Beijing 2008.

[12]. M.L.Tsai, K.W.Chiang, Y.W.Huang, Y.S. Lin, J.S. Tsai, C.F. Lo, Y.S. Lin, C.H. Wu, The Development of a Direct Georeferencing Ready UAV based photogrammetry platform, Commission I, ICWG I/V Unmanned Vehicle Systems (UVS) for Mapping and Monitoring Applications.

[13]. Henri Eisenbeiss, Martin Sauerbier (Zurich-Switzerland), Karsten Lambers(Bonn-German), Photogrammetric recording of the archaeological site of Pinchango Alto (Palpa, Peru) using a mini helicopter(UAV), Eisenbeiss_et_al_2007.

[14]. H. Bendea, F. Chiabrandoa, F. Giulio Tonolob, D. Marenchino, Mapping of archaeological areas using a low-cost UAV the AUGUSTA BAGIENNORUM test site, XXI International CIPA Symposium, 01-06 October 2007, Athens, Greece.

[15]. Anna Zmarz, Application of UAV in polish forestry to acquire image data, Polish association for spatial information annals of geomatics 2009, volume VII, number 2(32). 


\section{Summary}

\section{Building a topographical database from UAV imagery data}

\section{MSc. Do Van Duong}

\section{Ha Noi University of Natural Resource and Environment}

In the world, the unmanned aviation vehicle (UAV) have been widely applied in photogrammetry. The imagery data acquired from UAV can be used for both military and civilian purposes such as border supervision, natural hazard response, argriculture and

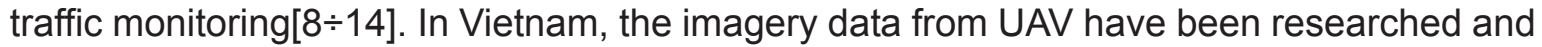

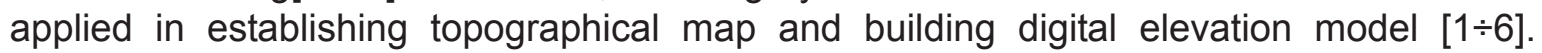
However, a Geographical database using UAV's imagery data is a new concept and is on the process of laboratory testing. This paper aims to describe the process of building a Geographical database from UAV's imagery data and analyze the performance of the result from field tests. $\bigcirc$

\section{XÂY DỰNG VÙNG GIÁ TRỊ ĐẤT.....}

\section{(Tiếp theo trang 24)}

[4]. Hồ Thị Lam Trà. "Định giá đất". Giáo trình trường Đại học Nông nghiệp I Hà Nội. 2005.

[5]. Roberto A. Figueroa. "Modeling the Value of Location in Regina Using GIS and Spatial Autocorrelation Statistics". Assessment Journal; Nov/Dec 1999; 6, 6; ABI/INFORM Global PP.29.

[6]. Bùi Ngọc Tuân (chủ trì, 2001), "Nghiên cứu một số nguyên nhân cơ bản làm biến động giá đất đô thị trên thị trường và đề xuất phương pháp xác định khung giá đất đô thị cho phù hợp", Tổng cục Địa chính.

[7]. Nguyễn Mạnh Hùng và nnk. "Phương pháp định giá bất động sản ứng dụng lý thuyết vị thế, chất lượng". O

\section{Summary}

\section{Building land value zone for non - agricultural land in urban by using statistical modeling and GIS technology}

\section{Dr. Nguyen Phi Son, Vietnam Institute of Geodesy and Cartography}

The functional setting of nominal land value based on multivariant recurrence analysis of factors forming land values at trading parcel of lands had been shown 78,24 percents of actual land costs. It means the surface of marking off the value will make sure the determination of land costs at any location with its accuracy of approximately 80 percents of the actual land costs on the market. Many facilities of model of value region which we had known. Among them, these applications to calculate the land costs of the market is very objective if the accuracy, and in timing of survey data, the allocation of survey position, particularly in trading amount of survey sampling, will make the quality of estimating model close to the fact. At the testing field of 5 wards of Viet Tri city, the model had been rendered that could determine the land costs at 78,24 percent of the actual price. At other regions, it may be evenly better if the data are adequate and accurate. This article was written on the research result of Ministry's theme "Research of mapping method for land value region of non-agricultural land area at the urban by statistical model and GIS technology" of code TNMT.07.31.O 